5th International Workshop on Astronomy and

Relativistic Astrophysics (IWARA2011)

International Journal of Modern Physics: Conference Series

Vol. 18 (2012) 211-215

(C) World Scientific Publishing Company

DOI: $10.1142 / \mathrm{S} 2010194512008471$

\title{
GLUEBALL-GLUEBALL POTENTIAL IN A CONSTITUENT GLUON MODEL
}

\author{
M. L. L. DA SILVA \\ Instituto de Física e Matemática, Universidade Federal de Pelotas \\ Caixa Postal 354, CEP 96010-090, Pelotas, RS, Brazil. \\ mario.silva@ufpel.edu.br \\ D. HADJIMICHEF \\ Instituto de Fúsica, Universidade Federal do Rio Grande do Sul \\ Caixa Postal 15051, CEP 91501-970, Porto Alegre, RS, Brazil. \\ dimiter.hadjimichef@ufrgs.br \\ C. A. Z. VASCONCELLOS \\ Instituto de Física, Universidade Federal do Rio Grande do Sul \\ Caixa Postal 15051, CEP 91501-970, Porto Alegre, RS, Brazil. \\ cesarzen10@hotmail.com
}

\begin{abstract}
In this work we use a mapping technique to derive in the context of a constituent gluon model an effective Hamiltonian that involves explicit gluon degrees of freedom. We study glueballs with two gluons using the Fock-Tani formalism. In the present work we calculate the glueball-glueball potential, in the context of the constituent gluon model, with gluon interchange.
\end{abstract}

Keywords: Glueball; potential; constituent model.

PACS numbers: 12.39.Mk,12.39.Pn, 12.39.Jh

\section{Introduction}

The gluon self-coupling in QCD implies the existence of bound states of pure gauge fields known as glueballs. Numerous technical difficulties have so far been present in our understanding of their properties in experiments, largely because glueball states can mix strongly with nearby $q \bar{q}$ resonances. In the present we follow a different approach by applying the Fock-Tani formalism in order to obtain an effective interaction between glueballs ${ }^{1}$ and then a glueball-glueball potential can be obtained. 


\section{The Fock-Tani Formalism}

The starting point in the present calculation is the definition, in second quantization, of the glueball creation operator formed by two constituent gluons

$$
G_{\alpha}^{\dagger}=\frac{1}{\sqrt{2}} \Phi_{\alpha}^{\mu \nu} a_{\mu}^{\dagger} a_{\nu}^{\dagger}
$$

Gluon creation $a_{\nu}^{\dagger}$ and annihilation $a_{\mu}$ operators obey the conventional commutation relations

$$
\left[a_{\mu}, a_{\nu}\right]=0 ;\left[a_{\mu}, a_{\nu}^{\dagger}\right]=\delta_{\mu \nu} .
$$

In (1) $\Phi_{\alpha}^{\mu \nu}$ is the bound-state wave-function for two-gluons. The composite glueball operator satisfy non-canonical commutation relations

$$
\left[G_{\alpha}, G_{\beta}\right]=0 \quad ; \quad\left[G_{\alpha}, G_{\beta}^{\dagger}\right]=\delta_{\alpha \beta}+\Delta_{\alpha \beta}
$$

where

$$
\delta_{\alpha \beta}=\Phi_{\alpha}^{\star \rho \gamma} \Phi_{\beta}^{\gamma \rho} \quad ; \quad \Delta_{\alpha \beta}=2 \Phi_{\alpha}^{\star \mu \gamma} \Phi_{\beta}^{\gamma \rho} a_{\rho}^{\dagger} a_{\mu} .
$$

The "ideal particles" which obey canonical relations, in our case are the ideal glueballs

$$
\left[g_{\alpha}, g_{\beta}\right]=0 \quad ; \quad\left[g_{\alpha}, g_{\beta}^{\dagger}\right]=\delta_{\alpha \beta} .
$$

This way one can transform the composite glueball state $|\alpha\rangle$ into an ideal state $\alpha)$ by

$$
\mid \alpha)=U^{-1}\left(-\frac{\pi}{2}\right) G_{\alpha}^{\dagger}|0\rangle=g_{\alpha}^{\dagger}|0\rangle
$$

where $U=\exp (t F)$ and $F$ is the generator of the glueball transformation given by

$$
F=g_{\alpha}^{\dagger} \tilde{G}_{\alpha}-\tilde{G}_{\alpha}^{\dagger} g_{\alpha}
$$

with

$$
\tilde{G}_{\alpha}=G_{\alpha}-\frac{1}{2} \Delta_{\alpha \beta} G_{\beta}-\frac{1}{2} G_{\beta}^{\dagger}\left[\Delta_{\beta \gamma}, G_{\alpha}\right] G_{\gamma} .
$$

In order to obtain the effective glueball-glueball potential one has to use (6) in a set of Heisenberg-like equations for the basic operators $g, \tilde{G}, a$

$$
\frac{d g_{\alpha}(t)}{d t}=\left[g_{\alpha}, F\right]=\tilde{G}_{\alpha} \quad ; \quad \frac{d \tilde{G}_{\alpha}(t)}{d t}=\left[\tilde{G}_{\alpha}(t), F\right]=-g_{\alpha} .
$$

The simplicity of these equations are not present in the equations for $a$

$$
\begin{aligned}
\frac{d a_{\mu}(t)}{d t}=\left[a_{\mu}, F\right]= & -\sqrt{2} \Phi_{\beta}^{\mu \nu} a_{\nu}^{\dagger} g_{\beta}+\frac{\sqrt{2}}{2} \Phi_{\beta}^{\mu \nu} a_{\nu}^{\dagger} \Delta_{\beta \alpha} g_{\beta} \\
& +\Phi_{\alpha}^{\star \mu \gamma} \Phi_{\beta}^{\gamma \mu^{\prime}}\left(G_{\beta}^{\dagger} a_{\mu^{\prime}} g_{\beta}-g_{\beta}^{\dagger} a_{\mu^{\prime}} G_{\beta}\right) \\
& -\sqrt{2}\left(\Phi_{\alpha}^{\mu \rho^{\prime}} \Phi_{\rho}^{\mu^{\prime} \gamma^{\prime}} \Phi_{\gamma}^{\star \gamma^{\prime} \rho^{\prime}}\right. \\
& \left.+\Phi_{\alpha}^{\mu^{\prime} \rho^{\prime}} \Phi_{\rho}^{\mu \gamma^{\prime}} \Phi_{\gamma}^{\star \gamma^{\prime} \rho^{\prime}}\right) G_{\gamma}^{\dagger} a_{\mu^{\prime}}^{\dagger} G_{\beta} g_{\beta} .
\end{aligned}
$$


The solution for these equation can be found order by order in the wave-functions. For zero order one has $a_{\mu}^{(0)}=a_{\mu}$

$$
\begin{aligned}
g_{\alpha}^{(0)}(t) & =G_{\alpha} \sin t+g_{\alpha} \cos t \\
G_{\beta}^{(0)}(t) & =G_{\beta} \cos t-g_{\beta} \sin t
\end{aligned}
$$

In the first order $g_{\alpha}^{(1)}=0, G_{\beta}^{(1)}=0$ and

$$
a_{\mu}^{(1)}(t)=\sqrt{2} \Phi_{\beta}^{\mu \nu} a_{\nu}^{\dagger}\left[G_{\beta}^{(0)}-G_{\beta}\right] .
$$

For more details about this calculation see $\operatorname{Ref}^{1}$. The glueball-glueball potential can be obtained applying in a standard way the Fock-Tani transformed operators to the microscopic Hamiltonian

$$
\mathcal{H}(\mu \nu ; \sigma \rho)=T_{\mathrm{aa}}(\mu) a_{\mu}^{\dagger} a_{\mu}+\frac{1}{2} V_{\mathrm{aa}}(\mu \nu ; \sigma \rho) a_{\mu}^{\dagger} a_{\nu}^{\dagger} a_{\rho} a_{\sigma}
$$

where in this microscopic Hamiltonian $T_{\text {aa }}$ is the kinetic energy and $V_{\text {aa }}$ is the potential in the constituent model. After transforming $\mathcal{H}(\mu \nu ; \sigma \rho)$ one obtains for the glueball-glueball potential $V_{g g}$

$$
V_{g g}=\sum_{i=1}^{4} V_{i}(\alpha \gamma ; \delta \beta) g_{\alpha}^{\dagger} g_{\gamma}^{\dagger} g_{\delta} g_{\beta}
$$

and

$$
\begin{aligned}
& V_{1}(\alpha \gamma ; \delta \beta)=2 V_{a a}(\mu \nu ; \sigma \rho) \Phi_{\alpha}^{\star \mu \tau} \Phi_{\gamma}^{\star \nu \xi} \Phi_{\delta}^{\rho \xi} \Phi_{\beta}^{\sigma \tau} \\
& V_{2}(\alpha \gamma ; \delta \beta)=2 V_{a a}(\mu \nu ; \sigma \rho) \Phi_{\alpha}^{\star \mu \tau} \Phi_{\gamma}^{\star \nu \xi} \Phi_{\delta}^{\rho \tau} \Phi_{\beta}^{\sigma \xi} \\
& V_{3}(\alpha \gamma ; \delta \beta)=V_{a a}(\mu \nu ; \sigma \rho) \Phi_{\alpha}^{\star \mu \nu} \Phi_{\gamma}^{\star \lambda \xi} \Phi_{\delta}^{\sigma \lambda} \Phi_{\beta}^{\rho \xi} \\
& V_{4}(\alpha \gamma ; \delta \beta)=V_{a a}(\mu \nu ; \sigma \rho) \Phi_{\alpha}^{\star \mu \xi} \Phi_{\gamma}^{\star \nu \lambda} \Phi_{\delta}^{\lambda \xi} \Phi_{\beta}^{\rho \sigma} .
\end{aligned}
$$

The scattering $T$-matrix is related directly to Eq. (7)

$$
T(\alpha \beta ; \gamma \delta)=\left(\alpha \beta\left|V_{g g}\right| \gamma \delta\right) \text {. }
$$

Due to translational invariance, the $T$-matrix element is written as a momentum conservation delta-function, times a Born-order matrix element, $h_{f i}$ :

$$
T(\alpha \beta ; \gamma \delta)=\delta^{(3)}\left(\vec{P}_{f}-\vec{P}_{i}\right) h_{f i}
$$

where $\vec{P}_{f}$ and $\vec{P}_{i}$ are the final and initial momenta of the two-glueball system. This result can be used in order to evaluate the glueball-glueball potential

$$
V_{G G}(r)=\int d^{3} Q e^{i \vec{Q} \cdot \vec{r}} h_{f i}(\vec{Q}) .
$$

The scattering amplitude $h_{f i}$ can be visualized in Fig. 1. 

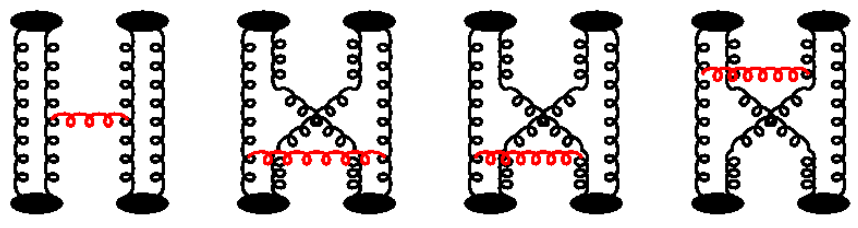

Fig. 1. Diagrams representing the scattering amplitude $h_{f i}$ for glueball-glueball interaction with constituent gluon interchange.

The potential $V_{\text {aa }}$ is determined in the Cornwall and Soni constituent gluon model $^{2}$

$$
V_{\text {aa }}(r)=\frac{1}{3} f^{a c e} f^{b d e}\left[V_{2 g}^{O G E P}(r)+V_{S}(r)\right]
$$

where

$$
V_{2 g}^{O G E P}(r)=-\lambda\left[\omega_{1} \frac{e^{-m r}}{r}+\omega_{2} \frac{\pi}{m^{2}} D(r)\right] \quad, \quad V_{S}(r)=2 m\left(1-e^{-\beta m r}\right)
$$

and

$$
D(r)=\frac{k^{3} m^{3}}{\pi^{3 / 2}} e^{-k^{2} m^{2} r^{2}} \quad, \quad \lambda=\frac{3 g^{2}}{4 \pi} \quad, \quad \omega_{1}=\frac{1}{4}+\frac{1}{3} \vec{S}^{2} \quad, \quad \omega_{2}=1-\frac{5}{6} \vec{S}^{2}(1
$$

The parameters $\lambda, m, k$ and $\beta$ assume known values ${ }^{3,4,5}$ while the wave function $\Phi_{\alpha}^{\mu \nu}$ is given $i n^{1}$. The glueball-glueball scattering amplitude $h_{f i}$ is given by

$$
h_{f i}(s, t)=\frac{3}{8} R_{0}(s) \sum_{i=1}^{6} R_{i}(s, t)
$$

where

$$
\begin{aligned}
& R_{0}=\frac{4}{(2 \pi)^{3 / 2} b^{3}} \exp \left[-\frac{1}{2 b^{2}}\left(\frac{s}{4}-M_{G}^{2}\right)\right] \\
& R_{1}=\frac{\lambda \omega_{1}^{(2)} 4 \sqrt{2 \pi}}{3} \int_{0}^{\infty} d q \frac{q^{2}}{q^{2}+m^{2}} \exp \left(-\frac{q^{2}}{2 b^{2}}\right)\left[\mathcal{J}_{0}\left(\frac{q \sqrt{t}}{2 b^{2}}\right)+\mathcal{J}_{0}\left(\frac{q \sqrt{u}}{2 b^{2}}\right)\right], \\
& R_{2}=\frac{\lambda \omega_{2}^{(2)} 2 \sqrt{2} \pi b^{3} k^{3} m}{3\left(b^{2}+2 k^{2} m^{2}\right)^{3 / 2}}\left[\exp \left(-\frac{t k^{2} m^{2}}{4\left(b^{4}+2 b^{2} k^{2} m^{2}\right)}\right)+\exp \left(-\frac{u k^{2} m^{2}}{4\left(b^{4}+2 b^{2} k^{2} m^{2}\right)}\right)\right], \\
& R_{3}=\frac{32 \sqrt{2 \pi}}{3} \int_{0}^{\infty} d q \frac{q^{2} \beta m^{2}}{\left(q^{2}+\beta^{2} m^{2}\right)^{2}} \exp \left(-\frac{q^{2}}{2 b^{2}}\right)\left[\mathcal{J}_{0}\left(\frac{q \sqrt{t}}{2 b^{2}}\right)+\mathcal{J}_{0}\left(\frac{q \sqrt{u}}{2 b^{2}}\right)\right] \\
& R_{4}=-\frac{\lambda \omega_{1}^{(3)}}{3} \frac{16 \sqrt{2 \pi} b^{2}}{\sqrt{\frac{s}{4}-M_{G}^{2}}} \int_{0}^{\infty} d q \frac{q}{q^{2}+m^{2}} \exp \left(-\frac{3 q^{2}}{8 b^{2}}\right) \sinh \left(\frac{q}{2 b^{2}} \sqrt{\frac{s}{4}-M_{G}^{2}}\right),
\end{aligned}
$$




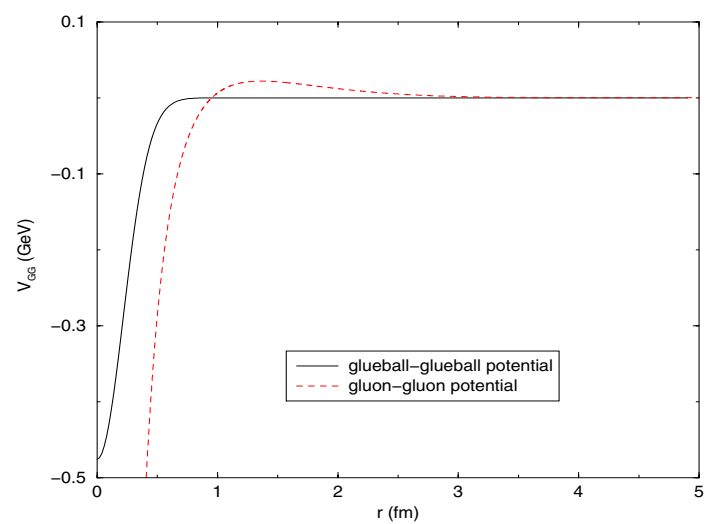

Fig. 2. Glueball-glueball and gluon-gluon potential for $0^{++}$with the following parameters $r_{0}=$ $0.58 \mathrm{fm}, \beta=0.1, \lambda=1.8, k=0.21$, gluon mass $m=0.6 \mathrm{GeV}$. The $s \bar{s}$ quark model parameters: $m_{q}=0.55 \mathrm{GeV}, \alpha_{s}=0.6$.

$$
\begin{aligned}
& R_{5}=-\frac{\lambda \omega_{2}^{(3)}}{3} \frac{16 \pi b^{3} k^{3} m}{\left(2 b^{2}+3 k^{2} m^{2}\right)^{3 / 2}} \exp \left[-\frac{k^{2} m^{2}\left(\frac{s}{4}-M_{G}^{2}\right)}{2\left(2 b^{4}+3 b^{2} k^{2} m^{2}\right)}\right], \\
& R_{6}=-\frac{128 \sqrt{2 \pi} b^{2}}{3 \sqrt{\frac{s}{4}-M_{G}^{2}}} \int_{0}^{\infty} d q \frac{q \beta m^{2}}{\left(q^{2}+\beta^{2} m^{2}\right)^{2}} \exp \left(\frac{3 q^{2}}{8 b^{2}}\right) \sinh \left(\frac{q}{2 b^{2}} \sqrt{\frac{s}{4}-M_{G}^{2}}\right) .
\end{aligned}
$$

Here $b=\frac{\sqrt{3}}{\sqrt{2} r_{0}}$ where $r_{0}$ is the glueball's rms radius and $\mathcal{J}_{0}(x)=\sin x / x$. In $(16)$ one finds the following notation $\omega_{1}^{(i)}$ and $\omega_{2}^{(i)}$, where the index $i$ corresponds to the number of the evaluated diagram in Fig. 1. The glueball-glueball potential is shown in the Fig. 2.

The glueball-glueball potential is a short range and deep potential. However, it is interesting to investigate if the gluenall glueball potential will form bound states.

\section{Acknowledgments}

This work was partially supported by FAPERGS and CNPq.

\section{References}

1. M. L. L. da Silva, D Hadjimichef, C. A. Z Vasvoncellos and B. E. J. Bodmann, J. Phys. $G, \mathbf{3 2}, 475$ (2006).

2. J. M. Cornwall and A. Soni, Phys. Lett. B120, 431 (1983).

3. W. S. Hou and A. Soni, Phys. Rev. D29, 101 (1984).

4. W. S. Hou, C. S. Luo and G. G. Wong, Phys. Rev. D64, 014028 (2001).

5. W. S. Hou and G. G. Wong, Phys. Rev. D67, 034003 (2003). 